\title{
Pulling Knotted Polymers
}

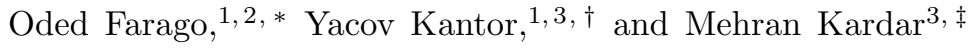 \\ ${ }^{1}$ School for Physics and Astronomy, Tel Aviv University, Tel Aviv 69978, Israel \\ ${ }^{2}$ Materials Research Laboratory, University of California, Santa Barbara, CA 93106, USA \\ ${ }^{3}$ Department of Physics, Massachusetts Institute of Technology, Cambridge, Massachusetts 02139, USA
}

\begin{abstract}
We compare Monte Carlo simulations of knotted and unknotted polymers whose ends are connected to two parallel walls. The force $f$ exerted on the polymer is measured as a function of the separation $R$ between the walls. For unknotted polymers of several monomer numbers $N$, the product $f N^{\nu}$ is a simple function of $R / N^{\nu}$, where $\nu \simeq 0.59$. By contrast, knotted polymers exhibit strong finite size effects which can be interpreted in terms of a new length scale related to the size of the knot. Based on this interpretation, we conclude that the number of monomers forming the knot scales as $N^{t}$, with $t=0.4 \pm 0.1$.
\end{abstract}

PACS numbers: 36.20.Ey 87.15.La 02.10.Kn

Entanglements are unavoidable in long polymers and influence their properties 䢗. Knots are found in proteins [2], and present an obstacle that needs to be overcome in the transcription of DNA [3]. An increasing number of experiments can now probe the detailed properties of knotted molecules [4]. Micro-manipulation techniques [5] enable direct measurements of mechanical properties of a single molecule, and it is even possible to probe the behavior of artificially knotted DNA [6]. However, incorporation of topological constraints into the statistical mechanics of polymers [7] remains a difficult theoretical challenge since the resulting partition of phase space into accessible and inaccessible regions cannot be easily implemented. Nevertheless, some progress has been made in understanding the role of knots in loop polymers; e.g. the relative probabilities for appearance of different knots in self-avoiding (SA) loops [8 has been characterized. However, much less is known about the typical shapes and physical properties of knots.
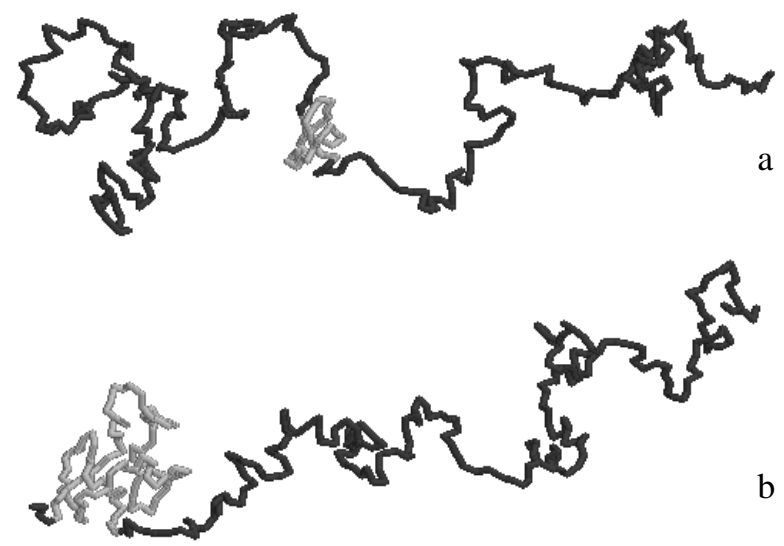

*Electronic address: farago@mrl.ucsb.edu

${ }^{\dagger}$ Electronic address: kantor@orion.tau.ac.i

${ }^{\ddagger}$ Electronic address: kardar@mit.edu
FIG. 1: Two configurations of a knotted chain of $N=335$ bonds, with its ends attached to parallel walls separated by $50 \mathrm{a}$. The walls are not shown and the chains were rotated for clarity of view. The (grey) knotted regions consist of 32 bonds in (a) and 112 bonds in (b).

As a topological feature, a knot can be rigorously identified only by specifying the entire shape of a closed chain (ring). However, it is natural to identify a segment where the knot is located, and consequently to talk about its size and statistics. It is natural to pull on the ends of a string to see if a small knot remains in the middle. Our eyes tend to identify knotted segments in this manner, as exemplified by the grey-shaded monomers of the polymers in Fig. 1. While pulling on a string makes tight knots, the question of whether in a random unforced chain the knot is spread over the whole curve, or localized (tight) on a small portion, is still not fully resolved. Several recent works [9] show that knots in SA walks confined to a two-dimensional (2D) plane are strongly localized, i.e. the mean number of monomers in the knotted region $N_{k}$ does not depend on the total number of monomers $N$. Studies of three-dimensional (3D) knotted polymers are hampered by the difficulty of identifying the knotted region. Katritch et al. [10 examined the size distribution of knots in 3D random rings, by removing segments of the ring, attaching them to infinite straight lines, and checking if the resulting structures were knotted. Although the method has a certain probability to fail (i.e., the procedure itself may create or remove a knot), it nevertheless suggests that knots in such rings are localized. By contrast, some studies [11] had earlier indicated that for moderate $N$, the radius of gyration $R_{g}$ of a knot is strongly influenced by its complexity, leading to the conclusion that the knots might be spread out over the entire loop [12]. More recent numerical results [13, 14] provide evidence that $R_{g}$ is asymptotically independent of the knot type, hinting that knots are localized.

Since correlation functions of SA walks are power laws, it is reasonable to expect that the distribution of the 
number of monomers $n$ forming a knot is also a power law, i.e. $p(n)=C n^{t-2}$. For $t<1$ the normalization coefficient $C$ is determined by the microscopic (short distance) properties of the chain, while for $0<t<1$, the expectation value of the mean knot size $N_{k}$ depends on the total length, growing as $N^{t}$. In such a case, we say that the knot is weakly localized. For $t<0$, the knot is strongly localized in the sense that $N_{k}$ is determined by the microscopic cutoff. In this paper, we attempt to quantify the tightness of knots in $3 \mathrm{D}$ polymers by comparing the force-extension relations of knotted and unknotted chains. As in Ref. [14, we find similarities between the statistical properties of knotted chains and those of shorter unknotted chains, a consequence of the fact that knotted segments are statistically denser than unknotted ones. Using the reduction in the effective number of monomers as an operational definition of knot size, we find $N_{k} \sim N^{t}$, with $t=0.4 \pm 0.1$.

Much of the current understanding of the scaling properties of long polymers is based on renormalization group ideas 15. The $N \rightarrow \infty$ limit in polymers corresponds to approaching a fixed point similar to that describing criticality in thermal phase transitions. In particular, it can be shown that for a polymer with either free ends or forming a closed loop (without any further restrictions on topology)

$$
R_{g}=a N^{\nu} \Phi\left(\frac{N_{0}}{N}, \frac{N_{1}}{N}, \ldots\right) \simeq A a N^{\nu}\left[1-B N^{-\Delta}\right],
$$

where $a$ is a microscopic length-scale (of the order of monomer diameter or bond length), while $\nu \approx 0.59$ (in $3 \mathrm{D}$ ) is a system independent (universal) exponent. The function $\Phi$ includes corrections to the leading power-law due to irrelevant variables, which can be interpreted as additional length scales $\left\{N_{i}\right\}$. Keeping only the largest such correction for large $N$ leads to the second part of the above equation. The dimensionless constants $A$ and $B$ are again system specific, while $\Delta \approx 0.5$ in $3 \mathrm{D}$ is universal [15]. If the leading correction comes from, say, the argument $N_{0} / N$ of $\Phi$, the corresponding length-scale grows as $N_{0} \sim N^{t_{0}}$, with $\Delta=1-t_{0}$.

For very long polymers, the correction term in Eq. (11) is irrelevant, and the dependence of many physical properties on the number of monomers $N$ can be cast in terms of a dependence on $R_{g}$ [16]. For example, consider the force $f$ needed to stretch a polymer between two parallel walls at a distance $R$. We can construct two dimensionless quantities, $f R_{g} / k_{B} T$ and $R / R_{g}$ ( $T$ is the temperature and $k_{B}$ is the Boltzmann constant), which must be functionally related. It is thus convenient to introduce variables $f^{\prime} \equiv f a N^{\nu} / k_{B} T$ and $R^{\prime} \equiv R / a N^{\nu}$, and express the force-extension relation in the form

$$
f^{\prime}=G\left(R^{\prime}\right) .
$$

Simple arguments [16] can now be used to determine the asymptotic behaviors of $G\left(R^{\prime}\right)$ : For a large stretching force, the distance $R$ between the ends of the polymer must be proportional to $N$. Conversely, in a strongly compressed state $\left(R \ll R_{g}\right)$, the force must be proportional to $N$. These limiting behaviors can be reconciled with Eq. (2) only if [16]

$$
G\left(R^{\prime}\right) \sim \begin{cases}R^{\prime \nu /(1-\nu)} & \text { for } \mathrm{R}^{\prime} \gg 1, \\ -R^{\prime-1-1 / \nu} & \text { for } \mathrm{R}^{\prime} \ll 1 .\end{cases}
$$

In the large force regime this gives $R \sim$ $a N\left(f a / k_{B} T\right)^{(1-\nu) / \nu}=a N_{b}^{\nu}\left(N / N_{b}\right)$ (omitting dimensionless prefactors), with $N_{b} \equiv\left(k_{B} T / f a\right)^{1 / \nu}$. Thus, the polymer can be viewed as a linear sequence of $N / N_{b}$ blobs, each of size $a N_{b}^{\nu}$ and consisting of $N_{b}$ monomers [17. On length-scales smaller than the blob size, the external forces are not significant, while on the length-scales larger the polymer is essentially linear. (An analogous blob picture is also available for the compressed regime [16].)

We employed Monte Carlo (MC) simulations to measure such force-extension relations. Our model chains were composed of hard spheres of diameter $0.75 a$ connected by "tethers" restricting the distance between adjacent spheres to be smaller than $a$, with no additional energy costs. The end-monomers were fixed to two infinite parallel walls a distance $R$ apart. An elementary MC step consisted of an attempt to move a randomly chosen sphere a distance $0.16 a$ in a random direction. $(N$ such attempts constitute one MC time unit.) With such parameters the chain cannot cross itself, and its topology is preserved by the impenetrable walls. The force $f$ was calculated from the probability densities of contacts between spheres, and the probability densities of having stretched tethers, as described in Ref. [18].

We studied chains of lengths $N=225,335,500$, and 750 , in both unknotted (simply connected), and knotted (connected via a single trefoil) states [19]. Fig. 1 depicts two different configurations of the knotted chains, where the region in which the knot is "concentrated" has been lighter shaded. For each $R$ and $N$ our simulations lasted about $10^{8} \mathrm{MC}$ time units, which is considerably longer than the estimated Rouse relaxation time [20]. We verified that during the relaxation period the knot is able to diffuse from one side to the other. Open and closed symbols in Fig. 2 depict the results for unknotted and knotted chains, respectively.

It is important to note that the usual derivation of Eqs. (1)-(3) is not for a specified polymer topology, but rather for an ensemble of polymers that includes all possible topologies. However, relations of this type are also likely to be valid for polymers of fixed topology, albeit with a different scaling function in Eq. (11). The collapse of the data for unknotted polymers (open symbols) of different lengths in Fig. 2 confirms this expectation. Indeed, the quality of the collapse indicates that subleading corrections are negligble for $R^{\prime}<2$. We have taken advantage of this observation to construct an analytic fit to the scaling function $G$, as depicted by the solid line 
in this figure. For $R^{\prime}>2$ the polymer with $N=335$ forms more than six blobs, each containing less than 55 monomers. For such small blobs, finite size effects begin to appear, as we observe a roughly $10 \%$ deviation in the right-most data points in Fig. 2. Note that a deviation of this magnitude is expected from Eq. 1, with $B \sim 1$.

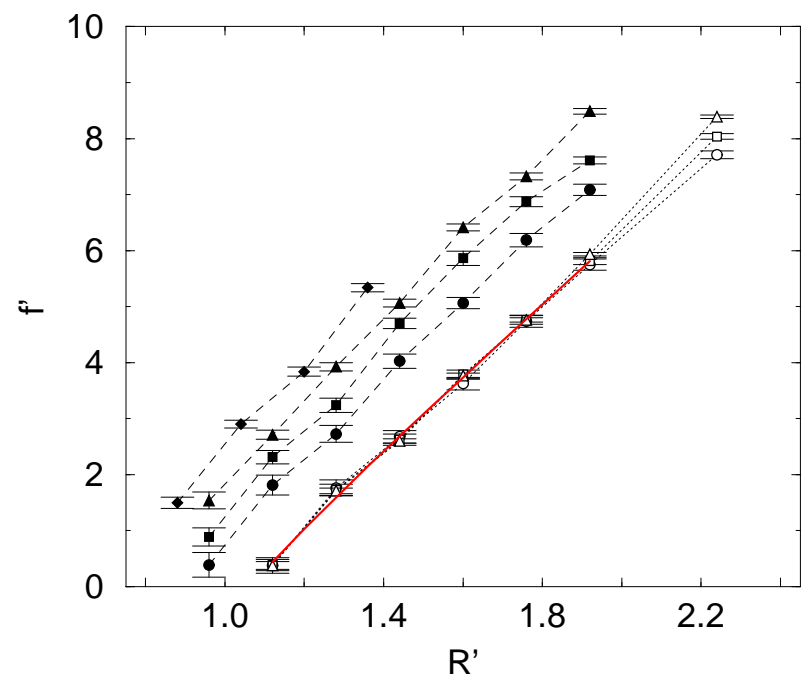

FIG. 2: The scaled force $f^{\prime}$ as a function of the scaled separation between walls $R^{\prime}$, for chains of sizes $N=750$ (circles), $N=500$ (squares), $N=335$ (triangles), and $N=225$ (diamonds) monomers. Open and solid symbols correspond, respectively, to unknotted and knotted chains. The solid line depicts an analytical fit to $G$ in this range.

As expected, for a given $f^{\prime}$ the knotted polymer (solid symbols in Fig. 2) has a significantly smaller $R^{\prime}$ than its unknotted counterpart. However, the scaled difference becomes less pronounced for larger $N$, and the results for longer chains approach those of the unknotted chain. The absence of data collapse indicates the appearance of strong finite size corrections in a parameter range where the unknotted chains show no such effect. We would like to associate this feature with the emergence of a new size scale due to the knot. If a string with a knot is fully stretched, its maximal length is reduced by the size of the resulting tight knot. While the knots in our simulations are far from tight, we shall still describe the influence of the knot as a reduction in the number of monomers $N$ by the 'size of the knot' $N_{k}$. If the knotted chain of length $N$ is equivalent to an unknotted chain of length $N-N_{k}$, its force-extension curves must satisfy $f a\left(N-N_{k}\right)^{\nu} / k_{B} T=G\left[R / a\left(N-N_{k}\right)^{\nu}\right]$, where $G$ is the scaling function obtained before for unknotted polymers (solid line in Fig. 2). Naturally, this definition will not work with a single $N_{k}$, since our knots are not tight. However, each solid data point in Fig. 2 can be moved to the previously obtained solid line by choosing an appropriate $N_{k}\left(f^{\prime}, N\right)$. This can be regarded as our operational definition of the size of the knot for a given $f$ and $N$.

If the force-extension curves for knotted polymers are to be consistent with the standard finite size corrections discussed in connection with Eq. (11), we must have

$$
f^{\prime}=G_{k}\left(R^{\prime}, \frac{N_{0}}{N}, \ldots\right) \simeq G\left(R^{\prime}\right)\left[1+g\left(R^{\prime}\right) N^{-\Delta_{k}}\right]
$$

Based on the numerical results, we have assumed that the leading scaling function $G$ is the same for knotted and unknotted polymers, but allowed for different corrections, such as a new exponent $\Delta_{k}$. For Eq. (A) to be consistent with our definition of knot size, we must have $N_{k}\left(f^{\prime}, N\right)=H\left(f^{\prime}\right) N^{t}$. The exponent $t=1-\Delta_{k}$ gives the scaling of the number of monomers in a knot (in the absence of force) via $N_{k} \sim N^{t}$. General scaling considerations do not restrict the shape of $H\left(f^{\prime}\right)$. However, from the "blob picture" of a strongly stretched chain, we know that the polymer is essentially linear beyond the blob size $N_{b}$ (and consequently not knotted on such scale), while within a blob it is undisturbed by the external forces. We thus expect the knot size to be determined by $N_{b}$ (as if this is the entire length of the polymer), i.e. $N_{k} \sim N_{b}^{t}=N^{t} / f^{\prime t / \nu}$, and $H\left(f^{\prime}\right) \sim 1 / f^{\prime t / \nu}$ for $f^{\prime} \gg 1$. Similar behavior occurs 21 in force-extension characteristics of polymers in which a sliding constriction (or slip-link 22]) creates a loop, somewhat reminiscent of a topological constraint. In the latter, the size of the loop under strong tension is equal to the size of a loop in unstressed polymers of size $N_{b}$.

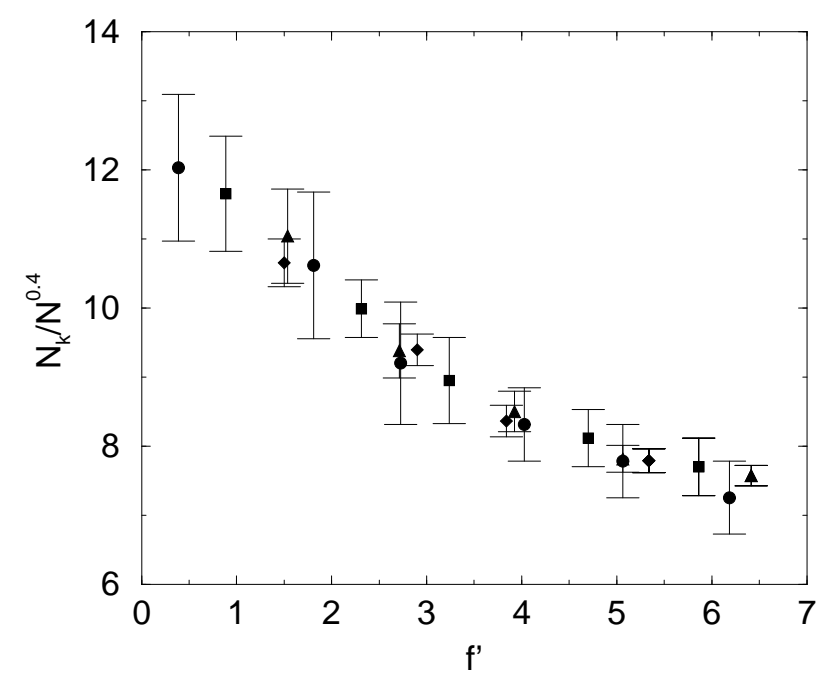

FIG. 3: The scaled knot size $N_{k} / N^{0.4}$, as a function of the scaled force $f^{\prime}$, for chain lengths $N=750$ (circles), 500 (squares), 335 (triangles), 225 (diamonds).

While our range of extensions is too limited to test the asymptotic behavior of $H\left(f^{\prime}\right)$, we can estimate the exponent $t$ from the value at which the functions $N_{k} / N^{t}$ exhibit the best collapse, i.e. are least sensitive to $N$. Fig. 3 depicts the optimal collapse with $t=0.4$, which is characterized by a $\chi^{2}$ value of 0.35 . For $t=0.3$ and $t=0.5$ we have $\chi^{2} \simeq 1$, serving as a criterion for the 
error in $t$, and our estimate of $t=0.4 \pm 0.1$. This is somewhat smaller than the value that can be deduced from Ref. [14. The corresponding finite-size correction exponent is $\Delta_{k}=0.6$. We note that this is close to the best numerical estimate $\Delta \simeq 0.56$, for the dominant correction to scaling when considering all topologies [23], and within errors, also is consistent with the estimate $\Delta \simeq 0.48$ obtained by field theoretic techniques 24. Is this more than simple coincidence? The standard field theory for polymers is based on an expansion around four dimensions that does not incorporate topological constraints. Assuming that the analytic continuation of this theory to three dimension also tells us about knots, how can such effects be anticipated in the perturbative expansion? If knots do indeed appear with subleading sizes in $3 \mathrm{D}$, their effects could be anticipated in corrections to scaling, in which case $\Delta_{k}=\Delta$. This conjecture is bolstered by $\Delta \simeq \Delta_{k} \simeq 1$ for knots confined to $2 \mathrm{D}[9,23]$.

In summary, by comparing force-extension relations of unknotted and knotted polymers of several lengths, we observe strong finite-size corrections in the latter, which we attribute to the knot size $N_{k}$. Scaling analysis and data collapse suggest a power law $N_{k} \sim N^{t}$, with $t=$ $0.4 \pm 0.1$. Thus unlike 2D 'flat knots' [9], the 3D knot sizes grow with the length of the polymer (although as a diminishing fraction of the whole length). Since a single knot is only weakly localized and "knows" about the size of the chain, it is interesting to investigate chains with several knots which may interact with each other. This, however, requires simulations with much larger chains.

We thank C. Jeppesen for helpful comments. Simulations were performed at High Performance Computing Unit of the Inter University Computation Center of Israel. This work was supported by the National Science Foundation (DMR-01-18213 and PHY99-07949) and the US-Israel Binational Science Foundation (1999-007). OF acknowledges the support of the MRL Program of the National Science Foundation (DMR00-80034).
[1] K. Mislow, Introduction to Stereochemistry, Benjamin, New York (1965); G. Schill, Catenanes, Rotaxanes, and Knots, Academic, New York (1971); D. M. Walba, Tetrahedron 41, 3161 (1985).

[2] W. R. Taylor, Nature 406, 916 (2000); M. L. Mansfield, Nature Struct. Biol. 1, 213 (1994); ibid. 4, 116 (1997); F. Takusagawa and K. Kamitori, J. Am. Chem. Soc. 118, 8945 (1996).

[3] B. Alberts, K. Roberts, D. Bray, J. Lewis, M. Raff and J. D. Watson, The molecular biology of the cell (Garland, New York, 1994).

[4] V. V. Rybenkov, N. R. Cozzarelli, and A. V. Vologodskii, Proc. Natl. Acad. Sci. USA 90, 5307 (1993); S. Y. Shaw and J. C. Wang, Science 260, 533 (1993).

[5] S. B. Smith, L. Finzi, and C. Bustamante, Science 258, 1122 (1992); B. Smith, Y. Cui, and C. Bustamante, Science 271, 795 (1996); K. Svoboda, S. M. Block, Ann. Rev. Biophys. Biomol. Structure 23, 247 (1994); A. Ashkin, Proc. Natl. Acad. Sci. USA 94, 4853 (1997); H. G. Hansma, J. Vac. Sci. Technology B14, 1390 (1995).

[6] Y. Arai, R. Yasuda, K.-I. Akashi, Y. Harada, H. Miyata, K. Kinosita Jr., and H. Itoh, Nature 399, 446 (1999).

[7] A. Yu. Grosberg and A. R. Khokhlov, Statistical Mechanics of Macromolecules (AIP Press, New York, 1994); A. L. Kholodenko and T. A. Vilgis, Phys. Rep. 298, 251 (1998); T. A. Vilgis, Phys. Rep. 336, 167 (1998).

[8] J. P. J. Michels and F. W. Wiegel, Phys. Lett. A 90, 381 (1982); D. W. Sumners and S. G. Whittington, J. Phys. A 21, 1689 (1988); E. J. Janse van Rensburg and S. G. Whittington, J. Phys. A 23, 3573 (1990); K. Koniaris and M. Muthukumar, Phys. Rev. Lett. 66, 2211 (1991); T. Deguchi and K. Tsurusaki, J. Knot Theory Ramif. 3, 321 (1994); M. K. Shimamura and T. Deguchi, Phys. Lett. A 274, 184 (2000); E. Orlandini, M. C. Tesi, E. J. Janse van Rensburg, and S. G. Whittington, J. Phys. A 29, L299 (1996); T. Deguchi and K. Tsurusaki, Phys. Rev. E 55, 6245 (1997).

[9] R. Metzler, A. Hanke, P. G. Dommersnes, Y. Kantor, and M. Kardar, preprint cond-mat/0110266 (2001); E.
Guitter and E. Orlandini, J. Phys. A 32, 1359 (1999); M. B. Hastings, Z. A. Daya, E. Ben-Naim, and R. E. Ecke, preprint cond-mat/0110612 (2001).

[10] V. Katritch, W. K. Olson, A. Vologodskii, J. Dubochet, and A. Stasiak, Phys. Rev. E 61, 5545 (2000).

[11] S. R. Quake, Phys. Rev. E 52, 1176 (1995); A. Yu. Grosberg, A. Feigel, and Y. Rabin, Phys. Rev. E 54, 6618 (1996).

[12] Force-size relations in small knots were studied in Y.J. Sheng, P.-K. Lai, and H.-K. Tsao, Phys. Rev. E61, 2895 (2000); P.-K. Lai, Y.-J. Sheng, and H.-K. Tsao, Macromol. Theor. Simul. 9, 578 (2000).

[13] E. J. Janse van Rensburg and S. G. Whittington, J. Phys. A 24, 3935 (1991).

[14] E. Orlandini, M. C. Tesi, E. J. Janse van Rensburg, and S. G. Whittington, J. Phys. A 31, 5953 (1998).

[15] J. des Cloizeaux and G. Jannink, Polymers in Solution, Clarendon Press, Oxford (1990); L. Schäfer, Excluded Volume Effects in Polymer Solutions, Springer, Berlin (1999).

[16] P. G. de Gennes, Scaling Concepts in Polymer Physics (Cornell University Press, Ithaca, 1979).

[17] P. Pincus, Macromol. 9, 386 (1976); P. G. de Gennes, P. Pincus, R. M. Velasco, and F. Brochard, J. Phys. (Paris) 37, 1461 (1976).

[18] O. Farago and Y. Kantor, Phys. Rev. E 61, 2478 (2000). To measure the force we use Eq.(14) in this paper with the prefactor $1 / V$ replaced by $1 / R$.

[19] Knots are traditionally defined on closed rings. To obtain an open chain with a trefoil one has to cut the ring at one of the arcs between two crossing points.

[20] M. Doi and S. F. Edwards, The Theory of Polymer Dynamics (Oxford University Press, Oxford, 1988).

[21] R. Metzler, Y. Kantor and M. Kardar, preprint (2002).

[22] M. Doi and S. F. Edwards, J. Chem. Soc. Faraday Trans. II 74, 1802 (1978); R. C. Ball, M. Doi, S. F. Edwards, and M. Warner, Polymer 22, 1010 (1981).

[23] B. Li, N. Madras, and A. D. Sokal, J. Stat. Phys. 80, 661 (1995). 
[24] R. Guida and J. Zinn-Justin, J. Phys. A 31, 8103 (1998). 